\title{
Case Report: Spontaneous Carinal Perforation and Bronchonodal Fistula due to Pulmonary Nocardiosis in an HIV-Infected Patient
}

\author{
Narongwit Nakwan* \\ Division of Pulmonology, Department of Medicine, Hat Yai Medical Education Center, Hat Yai Hospital, Songkhla, Thailand
}

\begin{abstract}
In clinical practice, nocardial infection is a rare opportunistic infection coexisting with potentially lifethreatening condition, particularly in patients with HIV infection. Although the incidence remains low, it is still associated with high morbidity and mortality. The lung is a typical site involved in this organism, and complicating conditions can be resulted from local disruption and or destruction of adjacent structures. To our knowledge, this is the first case of pulmonary nocardiosis in an HIV-infected patient who is complicated with spontaneous carinal perforation, leading to develop bronchonodal fistula.
\end{abstract}

\section{INTRODUCTION}

Nocardial infection is a rare opportunistic infection and a potentially life-threatening condition caused by Nocardia, which is a ubiquitous aerobic Gram-positive bacteria of the order Actinomycetales. ${ }^{1}$ Although incidence of this infection remains low, it is still associated with high morbidity and mortality. ${ }^{2}$ Nocardial infection usually infects individuals who have deficits in cell-mediated immunity such as transplant recipients, those receiving immunosuppressive therapy, and those with HIV infection, including those with chronic pulmonary disease. ${ }^{1}$ There are two clinical manifestations: localized and disseminated forms. However, the lung is usually primarily affected and be featured as pneumonic form. Furthermore, there can be multiple complicating conditions depending on the anatomic location of spreading disease, which can result in local disruption and or destruction of adjacent structures, including mediastinal lymphadenopathy, pleuropulmonary fistula, mediastinitis, empyema, endocarditis, and pericarditis. ${ }^{3}$ However, in the literature, carinal perforation leading to a bronchonodal fistula caused by Nocardia has not been previously reported. This report presents the first case of pulmonary nocardiosis in an HIV-infected patient, which resulted in spontaneous carinal perforation developing bronchonodal fistula.

\section{CASE REPORT}

A 19-year-old man presented with productive cough with greenish sputum and shortness of breath for 1 month. He also had a low-grade fever with night sweating. He was previously healthy with no history of trauma and was not currently on any medication. On examination, he had temperature of $38.5^{\circ} \mathrm{C}$ with blood pressure of $130 / 80 \mathrm{mmHg}$, heart rate of 110 beats/ minute, and respiratory rate of 30 breaths/minute. Pulse oximetry was $92 \%$ on room air. Examination revealed a thin, wellappearing young man in minimal distress. There were fine crepitation in the left lower lung field, dullness to percussion on the lower part of the left thorax, and normal heart sounds. There was no clubbing or lymphadenopathy. Oral thrush and oral hairy leukoplakia were observed in oral cavity. The rest of the physical examination revealed unremarkable results. The complete blood cell analysis showed a white blood cell of

\footnotetext{
* Address correspondence to Narongwit Nakwan, Division of Pulmonology, Department of Medicine, Hat Yai Medical Education Center, Hat Yai Hospital, 182 Rattakarn Road, Songkhla 90110, Thailand. E-mail: naronak@hotmail.com
}

$12.5 \times 10^{3}$ cell $/ \mu L$ with neutrophil predomination. Chest radiograph showed consolidation on the left lower lung area (Figure 1A). Computed tomography (CT) of the chest detected sub-carinal saccular air filling defect connecting to the carina surrounding with necrotic tissue together with consolidation on the entire left lower lung (Figure 1B). Sputum samples were tested for the acid-fast bacilli (AFB) and Gram stains, but all smears were negative. The assay Xpert MTB/ $\mathrm{RIF}^{\circledR}$ was tested, and the result showed no detection of Mycobacterium tuberculosis DNA. Subsequently, the fiberoptic bronchoscopy revealed carinal perforation at bifurcation of the carina connecting to the area of sub-carinal node with an irregular whitish necrotic border (Figure 1C). Several biopsies were obtained from the caseous tissue. Modified AFB stain on the bronchoalveolar lavage (BAL) demonstrated partially acid-fast beaded branching filaments, which was consistent with Nocardia spp. infection. However, the respiratory samples, including the necrotic tissue surrounding the carina, sputum, and BAL, were negative in the microbiological examinations for confirming the presence of Nocardia. The pathological findings were consistent with abscess with poorly formed granuloma with negative stains for AFB and modified AFB. The blood test for HIV antibody was detected, and a level of CD4 count was $16 \mathrm{cell} / \mu \mathrm{L}$. It was substantially diagnosed as pulmonary nocardiosis in HIV-infected patient with an incidental finding of spontaneous bronchonodal fistula on imaging and bronchoscopy. Trimethoprimsulfamethoxazole (TMP-SMX) was administered intravenously for 14 days. Topical antifungal therapy, clotrimazole, was administered for oral candidiasis. Then, the patient was discharged in a stable condition, and he was continued to take an oral form of TMP-SMX for next 12 months. Twelve weeks after taking antimicrobial medication, the patient was asked to reevaluate the carinal lesion under fiberoptic bronchoscopy and that lesion revealed a complete resolution of fistula. Also, chest radiograph after 12 weeks revealed near resolution on the left lower lobe. Although CT scan of the chest was recommended, he refused the advice because of financial problems. After the completion of 12-week treatment, he was sent to primary hospital for continuing the remaining course of treatment (totally 52 weeks) and starting antiretroviral therapy.

\section{DISCUSSION}

Tracheobronchial perforation has a significant association with either endotracheal intubation or blunt chest trauma, ${ }^{4}$ but 

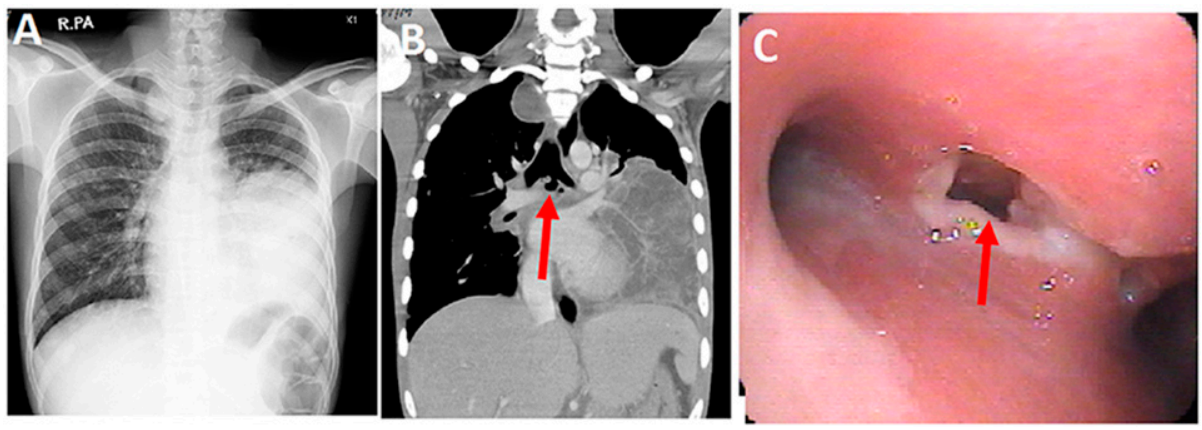

FIGURE 1. (A) Initial chest radiograph showed consolidation on the left lower lung area. (B) The initial chest computed tomography scan showed sub-carinal saccular air filling defect connecting to the carina surrounding with necrotic tissue (arrow) together with consolidation on the entire left lower lung. (C) Fiberoptic bronchoscopy revealed carinal perforation at bifurcation of the carina connecting to the area of subcarinal node with an irregular whitish necrotic border (arrow). This figure appears in color at www.ajtmh.org.

this condition seems to rarely occur in chronic pulmonary infection. However, there are a few published reports of spontaneous tracheal perforation developing bronchonodal fistula caused by tuberculosis infection, ${ }^{5-8}$ and such complication in pulmonary nocardiosis has never been reported in the literature. According to the epidemiology of HIV-related pulmonary infection, ${ }^{9}$ the patient's clinical presentations and radiographic findings in our case should primarily concern M. tuberculosis infection in differential diagnosis; however, all diagnostic studies for tuberculosis are negative. So, the nocardial infection was initially overlooked as a potential diagnosis due to low incidence organism among HIV-infected patients with chronic pulmonary infections. The clinical and radiological features in pulmonary nocardiosis are nonspecific, and microbiological diagnostic study is extremely difficult. ${ }^{10}$ For definite diagnosis, the culture of Nocardia is essential to confirm and subclassify species; however, this method is so complicated and unavailable in our hospital. To our case, the diagnosis was basically made by findings on modified AFB which demonstrated partially acidfast-beaded branching filaments from BAL; this method is quite easy, inexpensive, and available to general hospital. Based on acquired immunodeficiency syndrome-defining criteria, although HIV-related nocardiosis has not been classified in this criteria, ${ }^{11}$ most of the cases have occurred with the CD4 ${ }^{+}$ count less than 200 cell $/ \mu \mathrm{L}$ and more than $50 \%$ of them present as the first opportunistic infection in initially diagnosed as HIVinfected patients. ${ }^{2}$ To our case, the $\mathrm{CD} 4^{+}$count was $16 \mathrm{cell} / \mathrm{\mu L}$ that represented as such a immunocompromised status.

The diagnosis of carinal perforation in our patient can be very difficult because of nonspecific symptoms without any clue. Although mediastinal lymph node involved in pulmonary nocardiosis is not typical in either clinical or radiological features, our patient's condition might be resulted from rupturing necrotic sub-carinal lymph node infection into the carina which is a consequence of necrotizing mediastinitis from contiguous spread of infection from extensive consolidated lung. Nonetheless, it is important to note that the chest radiograph shows less benefit to detect this complication and may require $\mathrm{CT}$ imaging and/or bronchoscopy in patients with high suspicion for carinal perforation. For this reported patient, the chest CT demonstrated sub-carinal saccular air filling defect connecting to the carina surrounding with necrotic tissue coexisting with extensive consolidation on the left lower lung. These findings were compatible with carinal perforation as well as the findings on bronchoscopy.

With regard to the course of treatment, although there is a lack of prospective controlled trial and optimal antimicrobial treatment regimens have not been firmly established, the antimicrobial agent TMP-SMX remains the drug of choice for the treatment. ${ }^{1,10}$ However, alternative antimicrobial agents with activity against Nocardia, including amikacin, imipenem,

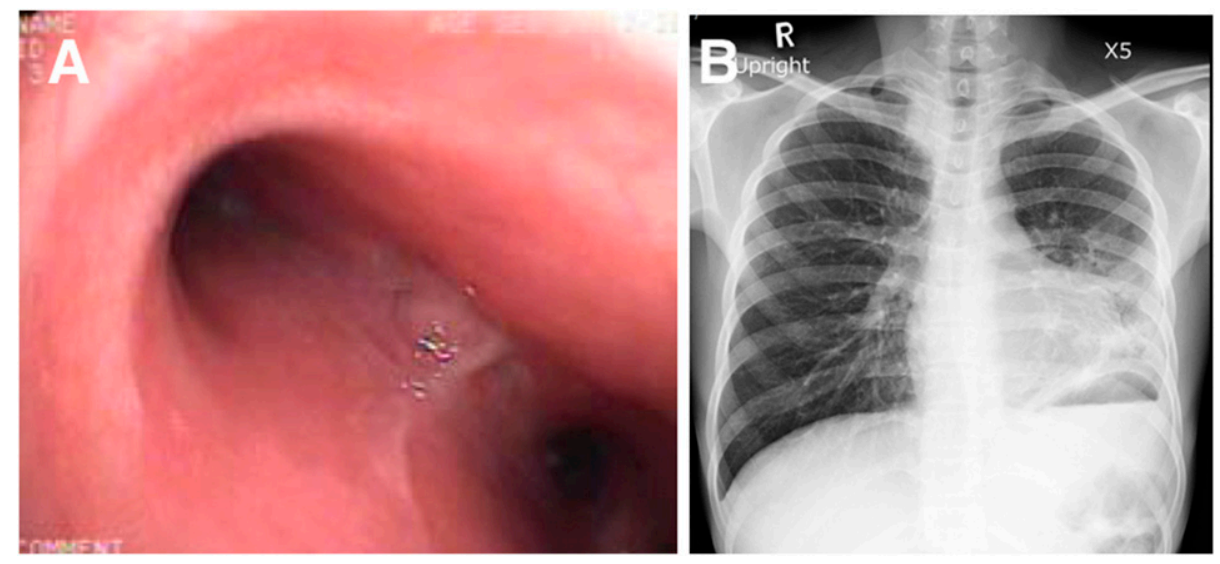

FIGURE 2. (A) Fiberoptic bronchoscopy 12 weeks after medical treatment showing a complete resolution of the fistula, and (B) chest radiograph revealing near resolution on the left lower lobe. This figure appears in color at www.ajtmh.org. 
minocycline, linezolid, and cephalosporins, have been recommended in a case of severe form or allergy to sulfonamide. ${ }^{11}$ The combination of amikacin and imipenem was more effective in the treatment of cerebral and pulmonary nocardiosis than TMP-SMX alone. ${ }^{12}$ The prognosis of pulmonary nocardiosis in the patients with HIV infection is poor, particularly in case of delayed diagnosis, extensive disease, and early discontinuation of treatment. ${ }^{10}$ The mortality remains high despite apparent response to medication. ${ }^{13}$ The duration of treatment should be least 12 months of antimicrobial therapy with the appropriate clinical monitoring especially in those with immunosuppression and those with CNS diseases. According to the treatment of carinal perforation, the conservative treatment with taking antimicrobial agent for a long term has been provided to the patient; however, surgical intervention would be considered by degree of infection and perforation. In our patient, his clinical response is well to those treatments, and such a lesion at the carina has complete resolution under repeated bronchoscopy after the 12-week treatment.

In conclusions, pulmonary nocardiosis has been considered in the HIV-infected patients with chronic pulmonary infection. Furthermore, spontaneous carinal perforation is a rare complication in pulmonary nocardiosis. Physicians should be aware, and early recognition is necessary because prognosis of patients with such complication remains very poor. However, conservative treatment with a long period of antimicrobial agent has been recommended in carinal perforation with bronchonodal fistula in nocardiosis.

Received October 9, 2020. Accepted for publication November 25, 2020.

Published online January 18, 2021.

Author's address: Narongwit Nakwan, Division of Pulmonology, Department of Medicine, Hat Yai Medical Education Center, Hat Yai Hospital, Songkhla, Thailand, E-mail: naronak@hotmail.com.

\section{REFERENCES}

1. Lerner PI, 1996. Nocardiosis. Clin Infect Dis 22: 891-905.

2. Pintado V, GÓmez-Mampaso E, Cobo J, Quereda C, Meswguer MA, FortÚn J, Navas E, Moreno S, 2004. Nocardial infection in patients infected with human immunodeficiency virus. Clin Microbiol Infect 9: 716-720.

3. Ambrosioni J, Lew D, Garbino J, 2010. Nocardiosis: updated clinical review and experience at a tertiarycenter. Infection 38: 89-97.

4. Grewal HS, Dangayach NS, Ahmad U, Ghosh S, Gildea T, Mehta AC, 2019. Treatment of tracheobronchial injuries. Chest 155: 595-604.

5. Lancella L, Nicolosi L, Bottero S, Carnevale E, Krzysztofiak A, Ticca F, 2003. Mediastinal tubercular lymphadenitis and adenobronchial fistulas (TABF) in the paediatric age. 1980-2001 case record. Infez Med 11: 75-80.

6. Park SH, Jeon KN, Park MJ, Bae K, Cho SB, Kim HC, Lee SJ, Cha $\mathrm{SI}, 2015$. Tuberculous bronchonodal fistula in adult patients: CT findings. Jpn J Radiol 33: 360-365.

7. Bae K, Jeon KN, Kim HC, Suh YS, Lee GD, Kim JY, Song DH, 2017. Tuberculosis presenting as isolated bronchonodal fistula in a patient with systemic lupus erythematosus: Case Rep Med (Baltimore) 96: e8764.

8. Liu J, Xie S, Yu L, Su X, 2019. Multidrug resistant lymph node fistula tracheobronchial tuberculosis: a case report. Medicine (Baltimore) 98: e18288.

9. Benito N, Moreno A, Miro JM, Torres A, 2012. Pulmonary infections in HIV-infected patients: an update in the 21st century. Eur Respir J 39: 730-745.

10. Uttamchandani RB, Daikos GL, Reyes RR, Fischl MA, Dickinson GM, Yamaguchi E, Kramer MR, 1994. Nocardiosis in 30 patients with advanced human immunodeficiency virus infection: clinical features and outcome. Cli Infect Dis 18: 348-353.

11. Saubolle MA, Sussland D, 2003. Nocardiosis: review of clinical and laboratory experience. J Clin Microbiol 41: 4497-4501.

12. Gombert ME, Berkowitz LB, Aulicino TM, duBouchet L, 1990. Therapy of pulmonary nocardiosis in immunocompromised mice. Antimicrob Agents Chemother 34: 1766-1768.

13. Sanchez-Munoz J, Yniguez TR, Garcia E, Pascua FJ, Crespo L, Bacaicoa A, Martín C, 1995. Nocardiosis in patients with human immunodeficiency virus infection in Spain. Rev Clin Esp 195: 468-472. 